Aus der geburtshülflich-gynäkologischen Klinik in Erlangen.

\title{
Zur Casuistik des conservativen Kaiserschnittes bei relativer Indication.
}

\author{
Von \\ Dr. med. Albert Döderlein, \\ I. Assistenzarzt.
}

(Mit 1 Curventafel.)

Durch die günstigen Resultate des conservativen Kaiserschnittes in den letzten Jahren auf Grund der Ausbildung sicherer Methoden erweiterten sich die Indicationen für denselben so sehr, dass jetzt in Fällen operirt wird, wo früher kaum Jemand es gewagt hätte, die Mutter eines so schweren Eingriffes zu unterziehen lediglich im Interesse des Kindes.

Folgender Fall möge als Illustration dazu dienen:

Frau Koch, Ausgehersfrau aus Erlangen, 24 Jahre alt, hat im Jahre 1883 in hiesiger Klinik zum ersten Male geboren. Die Geburt verlief äusserst schwer, da der Kopf das enge Becken unter langdauernden, kräftigsten Wehen sehr langsam passirte. Das Kind kam tief asphyktisch, konnte jedoch wiederbelebt werden.

Die Anamnese ergiebt: Patientin war stets gesund, hatte nur als Kind Masern und Scharlach; Laufen lernte sie angeblich mit einem Jahr. Menstruirt seit dem 14. Jahre regelmässig, vierwöchentlich, schmerzhaft. Letzte Periode Mitte April 1886. Ueber Conception, Kindsbewegungen weișs Patientin nichts anzugeben.

Am 27. Januar d. J. wurde ich Abends 11 Uhr zu Frau Koch gerufen, da die Geburt trotz kräftigster Wehen nicht weiterging. Wehen waren in rascher Folge und stetig steigender Heftigkeit seit $3 \mathrm{Uhr}$ Nachmittags, Blase Abends gegen $8 \mathrm{Uhr}$ gesprungen. Der Muttermund soll beim Blasensprunge verstrichen 
gewesen sein. Bei meiner Ankunft fand ich eine sehr kleine $(138 \mathrm{~cm})$ gracile, schwächliche Frau, die sich fortwährend stöhnend und schreiend im Bette umherwarf. Abdomen sehr stark, spitz aufgetrieben, Uterus dauernd hart, tetanisch contrahirt, Contractionsring deutlich einen Querfinger unter dem Nabel zu fühlen, schräg verlaufend, linkes Ligamentum rotundum straff gespannt. Kopf über dem Beckeneingange palpabel, sonst wegen der stetigen Contractionen keine Kindestheile zu fühlen. Herztöne links vom Nabel, laut, von normaler Frequenz. Aeussere Genitalien etwas geschwollen, Scheide ohne Besonderheit, vorliegender Theil Kopf, fest auf dem Beckeneingange, Kopfgeschwulst. Blase gesprungen, die etwa querfingerbreiten Muttermundsränder schlaff. Temperatur normal, Puls voll, nicht frequent.

Beckenmessung ergab:

Beckenumfang . . . . . . $76 \mathrm{~cm}$,

Distantia spinarum . . . . . 20,5,

" cristarum . . . . . . . 24 "

"trochanterum. . . . . 25 "

Conjugata externa B. . . . . 16,5 ",

$"$ diagonalis . . . . 10,

" vera geschätzt auf . . 8 ,

Allgemein verengtes, plattes Becken höchsten Grades.

Bei der beträchtlichen Verdünnung und Ausziehung des unteren Uterinabschnittes, beginnenden Druckerscheinungen, Erschöpfung der Kreissenden war eine baldige Entbindung geboten. Eine Spontangeburt war bei den räumlichen Missverhältnissen ausgeschlossen, ebensowenig war von einem Zangenversuche etwas zu hoffen, der die Prognose für das Kind nur gefährdet hätte; so blieb blos die Wahl zwischen Perforation des lebenden Kindes mit nachfolgender Kranioklasie oder Sectio caesarea.

Ich chloroformirte nun zunächst die Kreissende tief, bis der Uterus erschlaffte; um $1 / 21$ Uhr Morgens fuhren wir dieselbe in Narkose in die Klinik, wo nach wiederholter Untersuchung bei dem ganz hoch stehenden Kopfe der Entschluss zur Sectio caesarea gefasst wurde. Der Zustand änderte sich bis zum Beginn der Operation nicht. Die Wehen waren durch zeitweise Gaben von Morphium und Chloroform nicht mehr tetanisch, die Herztöne unverändert gut.

Die Vorbereitungen zur Operation bestanden in: Schwefeln des Operationszimmers, in dem bis zum Beginn der Operation und zum Theil auch während derselben zwei Dampfsprays 21/2 proc. 
Carbelnebel zerstäubten, Auskochen der Instrumente, die dann sofort in 5 proc. Carbolsäure gelegt wurden; von Seiten der Patientin in Entleerung der Blase, Rasiren der Pubes, Desinfection der Vagina und der Bauchdecken nach grünḍlicher Reinigung mit warmem Wasser und Seife mit $1 / 2000$ Sublimat.

Vor der Operation wurde die ganze Methode durchgesprochen, jedem Einzelnen seine bestimmte Function zugeschrieben, und zwar: ad manum assistirte dem Operateur, Herrn Prof. Zweifel, der I. Assistent, der II. Assistent Dr. Simon hatte zunächst die Aufgabe, bei Eröffnung der Uterushöhle durch Andrïcken von Schwämmen das Einfliessen von Blut und Fruchtwasser in die Bauchhöhle zu vermeiden, der eine Unterassistent chloroformirte, der andere reichte die Instrumente, die Oberhebamme sollte das Kind sofort nach der Extraction abnabeln und weiter versorgen.

Beginn der Narkose, Chloroform-Aether-Alkohol (100:30:30), um 5 Uhr Morgens.

1) Bauchschnitt in der Linea alba von Symphyse bis Processus xyphoideus, keine Blutung, Vorwälzung des leicht nach rechts gedrehten Uterus. Während nun Herr Prof. $Z$ weifel um das untere Ende des Uterus einen Schlauch anlegte und denselben zwar locker liess, aber doch die beiden Enden mit einer Billroth'schen Klammer zusammenpresste, um ihu im Moment einer starken Blutung zuzuschnüren, schloss ich die Bauchwunde oberhalb des Uterus mit zwei tiefgreifenden Seidennähten bis dicht an denselben.

2) Uterusschnitt in der Mitte. Zunächst wurde am Fundus die ganze Dicke durchtrennt, bis zwei Finger eingeschoben werden konnten, auf welchen der Schnitt in einem Zuge bis unten verlängert wurde, während dessen ich mit der rechten Hand einging, einen Fuss ergriff und das Kind extrahirte. Dasselbe war innerhalb drei Minuten nach Ansetzen des Messers an die Bauchdecken geboren und abgenabelt. Die Nabelschnur pulsirte kräftig, normal frequent. Das Kind schrie und athmete nicht gleich, der Zustand dürfte jedoch als Apnoë resp. Euapnoë, „Athemlosigkeit in prognostisch gutem Sinne“" im Gegensatz zur Asphyxie resp. Dysapnoë aufgefasst werden. Die kurze Zeit von Beginn der Operation bis zur Extraction, die normale Frequenz und Stärke der Herztöne bei Beginn derselben, der Nabelschnurpuls in normaler Frequenz und Stärke, der Umstand, dass die an der hinteren Wand sitzende Placenta durch den Schnitt nicht getroffen wurde, 
das Kind also keinen Tropfen Blut verlor, die normale Hautfarbe desselben, dies Alles lässt einen Scheintod, selbst leichten Grades; mit Sicherheit ausschliessen. Man sieht in diesem Falle, wie nahe beide Zustände in der Erscheinung sind, weil bei beiden keine Athmung existirt, trotzdem sie vollständig von einander verschieden sind. Im ersten Falle ist gar keine Gefahr für das Leben, im zweiten die allergrösste.

3) Die Blutung war beim Uterusschnitt, trotzdem derselbe die Placenta nicht traf, ziemlich heftig. Sofort nach Extraction des Kindes drängte sich die Placenta etwas vor, dieselbe wurde sammt den Eihäuten manuell ohne Schwierigkeit gelöst. Zugleich wurden Muttermund und Cervicalkanal sorgfalltig auf ihre Durchgängigkeit geprüft. Beim Abheben der Placenta von ihrer Haftstelle quoll zuerst gar kein Blut hervor. Die Wand war ganz weiss, zwischen Decidua serotina und der Uteruswand lag kein Blut. Einige Secunden später drang aus unzähligen Punkten der Blutstrom hervor und überschwemmte das Gesichtsfeld. Der Eintritt von Blut und Fruchtwasser in die Bauchhöhle wurde dadurch, dass die Banchwunde vor Eröffnung des Uterus bis an denselben geschlossen wurde, und durch seitliches Andrücken grosser Schwämme vermieden. Nun wurde der Schlauch fest angezogen, bis aus der Wunde und der Uterushöhle kein Tropfen Blut kam. Der Uterus wurde umgestuilpt und sorgfältig von allen Deciduafetzen durch Abschaben mit dem Fingernagel gereinigt; die Höhle sodann mit nassem Carbolschwamm ausgewischt und allenthalben Jodoform eingerieben. Naht der Wunde durch 14 tiefgreifende Nähte mit stärkster, geflochtener Seide ( $\mathrm{Nr} .14$ ) in der Weise, dass von innen nach aussen mit Freilassung der Decidua eingestochen und ca. $1 \mathrm{~cm}$ rom Wundrande der Serosa entfernt ausgestochen wurde, wozu natürlich an jedem Fadenende eine Nadel nöthig war. Nachdem diese Fäden, die erst nach Anlegen aller geknotet wurden, möglichst kurz geschnitten waren, wurde über denselben die Serosa in Falten (Lembert'sche Darmnaht) an einander genäht, und zwar durch 24 Knopfnähte mit Chromsäurecatgut (Nr. 2), so dass also im Ganzen 38 Nähte lagen. Die seroserösen Nähte schnitten erst im oberen Wundwinkel, wo angefangen wurde, durch; als sich jedoch der Uterus durch Contractionen verkleinerte, war das Peritoneum mit Leichtigkeit in Falten an einander $\mathrm{zu}$ bringen und schliesslich von den Seidennähten nichts mehr zu sehen. 
Das Anlegen der Muskulaturnähte erforderte: acht Minuten, der oberflächlichen 12 Minuten, so dass der Schlauch, da die Reinigung und Dosinfection etwa 5 Minuten in Anspruch nahm, nur ca. 25 Minuten angezogen war. Nach Lösung des Schlauches füllte sich der bis dahin völlig anämische Uterus allmälig, eine stärkere Blutansammlung wurde durch sofortige Massage und Auflage von Eisstïckchen, wodurch Contractionen $\mathrm{zu}$ erzielen waren, vermieden. Aus der Wunde kam nirgends Blut. Der Uterus contrahirte sich nun völlig gleichmässig und war namentlich schön die Anordnung der Uterusmuskulatur zu sehen. In der Mitte, von rückwärts gesehen, verlief eine Art Raphe oder Linea alba, von der nach beiden Seiten in der unteren Hälfte die Muskelfasern in zwei Bändern auseinandergingen. In der oberen Hälfte trafen dieselben zusammen, um am Fundus nach den Tuben hin auszustrahlen. ${ }^{2}$ )

\section{Verlauf:}

1. Tag. Erwacht fühlt sich Patientin wohl, geringe Nachwehen. Um $7 \mathrm{Uhr}$ geht Blut ab, intrauterine Ausspülung mit Eiswasser fördert grosse Blutcoagula zu Tage, Blutung steht, ein Mal Er-

28 Januar $1887=1$. Tag

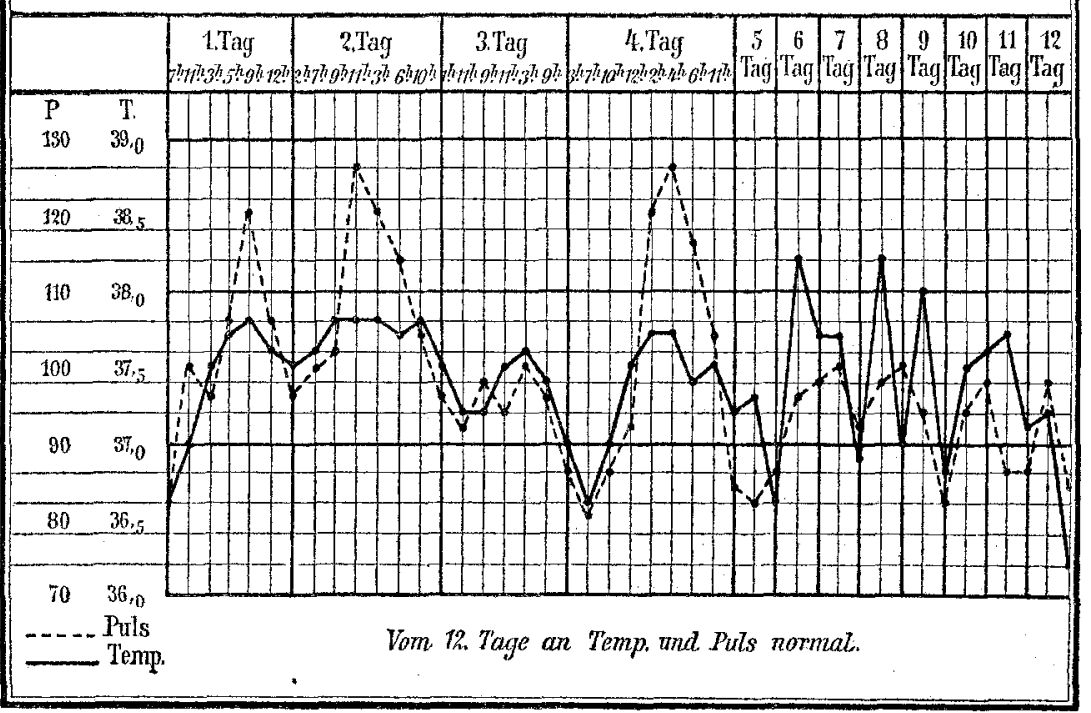

1) Vergl. die Abbildnng in Hunter's Atlas und Fig. 46 in $Z \mathrm{~W}$ eifel's Lehrbuch für Geburtshülfe (S, 76). 
brechen. Später gegen Schmerzen im Leibe 30 Tropfen Tinctura thebaicae, 1. Suppositorium (Opium 0,03). Eisblase auf den Leib. Puls voll, frequent.

2. Tag. Kein Erbrechen, Schmerzen im Leibe, mehrmals Suppositorium wiẹ oben, Abgang von Flatus, reichlicher Lochienabgang. Puls namentlich auf Opiumgabe frequent, doch voll.

3. Tag. Wohlbefinden, Bouillon mit Ei wird gut vertragen.

Der weitere Verlauf gestaltete sich, ausser geringen Eiterungen durch zwei Stichkanäle mit vorübergehender abendlicher Temperatursteigerung bis $38,2^{0}$, normal. Schon in den ersten Tagen war eine Temperatursteigerung um 0,2 bis $0,3^{\circ} \mathrm{C}$. und Pulsfrequenzsteigerung direct nach Gabe von Opium beobachtet; nach 10 Tagen wurde zur Sicherstellung dieser Beobachtung zwei Mal 30 Tropfen Tinctura Opii gegeben, worauf sich wieder Temperaturund Pulsfrequenzsteigerung zeigte, und zwar fand diese Steigerung in der ersten Stunde nach der Opiumdarreichung statt.

Dasselbe konnte auch ein drittes Mal in gleicher Weise beobachtet werden.

Die Wunde schloss sich in der Mitte nicht; eiternde Granulationen, die trotz Behandlung mit Lapis infernalis und Jodoform nicht heilten, wurden durch Abstossen der Uterusseidennähte unterhaiten. Ende März gelang es, mit dem Nahtsucher aus der Tiefe 3 geschlossene Fäden zu entfernen. Patientin scheint gegen Carbolseide besonders empfindlich zu sein, da auch zwei Bauchdeckenstichkanäle abscedirten, was wir bei den letzten 18 Laparatomirten nicht mehr zu beobachten Gelegenheit hatten. Herr Prof. $Z$ we ifel würde kommenden Falles die Uterusnaht überhaupt nur mit Chromsäurecatgut machen.

Das Kind, ein kräftig entwickelter Knabe, $50 \mathrm{~cm}$ lang, $3300 \mathrm{~g}$ schwer, litt in der ersten Zeit an Darmkatarrh, gedieh aber später sehr gut und hatte bei der Entlassung (19. März 1887) nahezu $500 \mathrm{~g}$ zugenommen. 